\title{
O ESTRESSE OXIDATIVO NA REGENERAÇÃO HEPÁTICA EM RATOS
}

\author{
OXIDATIVE STRESS ON LIVER REGENERATION IN RATS \\ José Ulisses de Souza Melo, TCBC-CE'; Manoel Messias Campos Júnior²; \\ Jefferson Menezes Viana Santos ${ }^{3}$; Marcus Vinicius Amaral Barreto ${ }^{3}$; Osamu de Sandes Kimura ${ }^{3}$
}

\begin{abstract}
RESUMO: Objetivo:. Investigar o estresse oxidativo durante a regeneração hepática em ratos submetidos à hepatectomia (HP) e, ao mesmo tempo, avaliar a função hepática enquanto em regeneração. Método: 36 ratos Wistar machos jovens foram aleatoriamente distribuídos em dois grupos de 18 animais: submetidos somente à laparotomia (controle, Grupo G1) e parcialmente hepatectomizados (experimento, Grupo G2). Nos tempos 36h (T1), 168h (T2) e 336h (T3) pós-HP, GSH foi medida no plasma e no tecido hepático, enquanto Gli e BT foram aquilatados no sangue. A massa do fígado residual foi utilizada para estimar a evolução da regeneração hepática. Resultados: Houve diferença estatisticamente significativa no crescimento dos lobos residuais nos grupos controle e experimento. GSH hepático e plasmático se mostraram significantemente maior nos animais parcialmente hepatectomizados.,em todos os tempos. Hiperglicemia estatisticamente significativa ocorreu nos ratos pós-HP nos tempos T2 e T3. A BT não apresentou qualquer alteração entre os grupos. Conclusão: Durante a regeneração hepática pós-HP em ratos há um aumento do estresse oxidativo e o fígado residual permanece apto na manutenção da homeostase orgânica (Rev. Col. Bras. Cir. 2008; 35(6): 411-415).
\end{abstract}

Descritores: Hepatectomia; Estresse oxidativo; Ratos; Regeneração hepática; Peroxidação de lipídeos; Glutationa.

\section{INTRODUÇÃO}

O fígado dos mamíferos possui uma intrigante capacidade de regeneração, o que já é conhecido desde há muito tempo e, no rato, após ressecção dos lobos lateral esquerdo e mediano (HP), os quais representam aproximadamente $67 \%$ da massa hepática total ${ }^{1}$, os lobos residuais, lateral direito e caudato, deflagram uma resposta essencialmente hiperplásica com regeneração de células e tecidos que culminam, em 3 a 14 dias, na restauração do volume original da glândula ${ }^{2,3,4}$. A peroxidação lipídica tem sido implicada como um dos fatores na enorme gama de influenciadores no fenômeno da regeneração $0^{5,6,7}$.

A glutationa (GSH), considerada como um dos componentes mais importantes do sistema defensivo antioxidante das células ${ }^{8}$ está presente em humanos, animais, plantas e bactérias aeróbias em altas concentrações ${ }^{9}$ e é abundante ( 3 a $10 \mathrm{mM}$ ) tanto no citoplasma, como no núcleo e na mitocôndria, tornando alta a probabilidade que espécies reativas de oxigênio (ERO) sejam eliminadas pela reação com GSH antes que possam iniciar os efeitos danosos. No plasma e em outros fluidos corporais são encontrados apenas traços de $\mathrm{GSH}^{10}$, exceto no trato respiratório inferior, onde sua função parece ser neutralizar as toxinas inaladas e os radicais livres produzidos pela ativação de macrófagos pulmonares. Na verdade, a glutationa, o maior constituinte antioxidante solúvel nos com- partimentos intracelulares ${ }^{11}$, é um termo coletivo para se referir ao tripeptídeo L-gama-glutamil-L-cisteinilglicina tanto em sua forma reduzida GSH, um monômero, como em sua forma dimérica oxidada GSSH, também chamada de diglutationa ou dissulfeto de glutationa. A ação antioxidante da GSH é facilitada pelo grupo sufidril da cisteína e, na oxidação, o enxofre forma o radical tiol que reage com uma segunda glutationa oxidada formando a ligação dissulfídrica (GS-SH).

São várias as maneiras que a GSH pode exercer sua função antioxidante: pode reagir quimicamente, por exemplo, com oxigênio singlete e com peróxidos (de hidrogênio e lipídicos), se classificando como varredora de radicais livres em sua forma direta, e pode estabilizar as estruturas das membranas pela remoção dos acil-peróxidos formados via peroxidação lipídica. Além disto, GSH é o agente redutor que recicla o ácido ascórbico de sua forma oxidada para sua forma reduzida pela enzima dehidroascorbato redutase. E, independente de suas ações antioxidantes, a GHS atua de modo direto ou indireto em muitos outros processos biológicos de suma importância dentre os quais estão a síntese de proteínas, o metabolismo, a desintoxicação por xenobióticos, os sinais de transducção, a expressão genética e a apoptose ${ }^{8,12}$. GSH não é um nutriente essencial visto que é sintetizada no fígado a partir dos aminoácidos glutamato, cisteína e glicina e, sob condições fisiológicas, o consumo de GSH tem taxa determinada pela sua utilização nos processos de detoxificação ${ }^{13}$.

1. Doutor em Cirurgia pela Universidade Federal do Ceará (UFC); M.Sc., NYU-USA; Mestre em Matemática pela UnB; Professor Associado do Departamento de Cirurgia da UFC.

2. Médico R1 em Cirurgia Geral, do Hospital Geral de Fortaleza - Ceará.

3. Acadêmico de Medicina da UFC.

Recebido em 04/08/2007

Aceito para publicação em 30/09/2008

Conflito de interesses: nenhum

Fonte de financiamento: nenhuma

Trabalho realizado no Laboratório de Cirurgia Experimental (LABCEX) do Programa de Pós-Graduação Stricto Sensu em Cirurgia da UFC e detentor do Prêmio Lacerda Machado 2008 ( $2^{\circ}$.lugar) da XVI Jornada Anual de Cirurgia da UFC. 
Baixas concentrações de GSH têm sido reportadas em algumas enfermidades, como a AIDS, e estão geralmente associadas a um maior risco de estresse oxidativo e da ocorrência de infecções oportunísticas.

A HP, gerando o fenômeno da regeneração hepática, ao se constituir em um trauma per se, induz um estresse oxidativo que, conforme sugerem as pesquisas, se constitui em fator incluso na enorme gama de fatores indutores, determinantes e influenciadores da regeneração $0^{5,6,7,14}$.

\section{MÉTODO}

A presente pesquisa obedeceu às normas do Colégio Brasileiro de Experimentação Animal (COBEA) e foi aprovada pela Comissão de Ética em Pesquisa Animal (CEPA) da UFC sob o número protocolar 14/06 de 11 de Agosto de 2006.

Foram utilizados 36 ratos machos, jovens ( $76 \pm 10$ dias), (Rattus norvegicus: var. albinus, Rodentia, mammalia) da linhagem Wistar, com peso médio de $142,65 \mathrm{~g}$ provenientes do Biotério Central da UFC e albergados, para o estudo, no biotério do LABCEX do Departamento de Cirurgia da UFC em gaiolas de prolipropileno com tampa de aço inoxidável e piso forrado com maravalhas, deixando aproximadamente $250 \mathrm{~cm}^{2}$ de área livre. Os animais foram mantidos em ciclos circadianos de claro/escuro, com 12 horas de luz de baixa intensidade e 12 horas de escuridão, tanto antes como após os procedimentos cirúrgicos e por todo o período da experimentação. As gaiolas foram higienizadas e os animais examinados cotidianamente. A temperatura ambiente foi mantida entre 18 e $27^{\circ} \mathrm{C}$, a umidade relativa do ar entre 40 e $70 \%$ e foi livre o acesso à água e à ração balanceada própria para a espécie composta de $4 \%$ de lipídios, $21 \%$ de proteínas, $52 \%$ de carboidratos e o restante de resíduos não digeríveis (Guabi Nutrilabor®, Mogiana Alimentos - São Paulo, S.P.)

Os animais foram distribuídos ao acaso em dois grupos de 18 ratos.

Grupo G1 - grupo controle - os animais foram submetidos somente à laparotomia no tempo T0 e, em cada um dos tempos T1(36h), T2(168h) e T3(336h) após a intervenção cirúrgica - laparotomia -, um lote de seis ratos deste grupo, tomados ao acaso, foram anestesiados e submetidos à relaparotomia, à coleta de sangue por punção da veia cava abdominal sob visão direta e, em seguida, à hepatectomia total (HT) e, em contínuo, sacrificados via dose letal inalatória de éter dietílico. O fígado foi então pesado e, para avaliação da $\mathrm{GSH}$, congelado a $-78^{\circ} \mathrm{C}$. O sangue foi coletado em dois tubos de ensaio: um para as aferições da glicose e da bilirrubina e outro para a mensuração da GSH plasmática.

Grupo G2, grupo teste, os ratos foram submetidos à hepatectomia parcial à $70 \%$ no tempo $\mathrm{T} 0 \mathrm{e}$, tal como no $\mathrm{G} 1$, em cada tempo T1, T2 e T3, 6 ratos escolhidos ao acaso foram relaparotomizados, com coleta de sangue e então submetidos à hepatectomia complementar (HC) e, a seguir, sacrificados. Os animais não foram submetidos a qualquer tipo de restrição (água, alimento etc.) antes dos procedimentos cirúrgicos.

No tempo T0, sob anestesia inalatória de éter dietílico, a laparotomia foi realizada via incisão transversa oblíqua subcostal bilateral com concavidade ligeiramente caudal com aproximadamente $4 \mathrm{~cm}$ de extensão. Nos animais G1, realizouse somente manipulação intra-abdominal (como se a HP fosse ser efetuada) por aproximadamente cinco minutos (tempo médio da HP) e síntese em seguida (nos mesmos moldes da síntese em G2 abaixo descrita). Nos ratos G2, após a abertura da parede abdominal, as alças intestinais foram afastadas, identificou-se o fígado e seus lobos, ressecção do ligamento teres hepatis e, em seguida, ligadura com fio de algodão zero do pedículo hepático composto pela veia porta, artéria hepática e via biliar, acima dos lobos posteriores (caudato e lateral direito). Em sequiência, foi feita a secção da tríade portal logo acima da ligadura, com conseqüente ressecção, em bloco, dos lobos anteriores, resultando em uma hepatectomia parcial bilobular de aproximadamente $70 \%$ conforme descrito por Higgins \& Anderson em $1931^{2}$. Realizadas as revisões da cavidade abdominal e da hemostasia, procedeu-se à síntese via laparorrafia em dois planos: o primeiro peritônio-músculo-aponeurótico com chuleio contínuo simples de categute 4.0 e o segundo, o da pele, com pontos separados usando fio de algodão 2.0. O animal foi deixado acordar pela suspensão do anestésico inalatório, permanecendo isolado em gaiola, com a oferta livre de água.

Nos tempos T1, T2 e T3, enquanto os animais de G1 foram submetidos à HT, os de G2 tiveram HC. Em ambos, antes da hepatectomia propriamente dita, realizou-se a colheita de sangue da veia cava por punção em sentido céfalo-caudal a $45^{\circ}$ com agulha hipodérmica $25 \times 7(0,7 \mathrm{~mm} \times 25 \mathrm{~mm}, 22 \mathrm{Gx} 1$ ", NIPRO ${ }^{\circledR}$, Sorocaba, SP) acoplada em seringa de $5 \mathrm{~mL}$ (BD Plastipak ${ }^{\circ}$, Curitiba, PR).

A glicemia e a bilirrubinemia serviram como parâmetros da avaliação do metabolismo hepático sob regeneração e as medidas do antioxidante GSH, tanto hepático como sangüíneo, para mensurar o estresse oxidativo. Para a determinação da GSH, calculou-se o teor dos grupos sulfidrílicos não protéicos pela técnica de Sedlak \& Lindsay ${ }^{15}$, o teor de bilrrubina total foi dosado via Meites ${ }^{16}$ e a D-glicose foi determinada segundo o método de Slein ${ }^{17}$.

A hiperplasia celular compensatória dos lobos residuais - a regeneração hepática - após hepatectomia a 70\% foi avaliada pela medidas das massas dos fígados residuais dos ratos nos tempos T1 (36h), T2 (168h) e T3 (336h) posteriores à amputação cirúrgica parcial do fígado. Tais mensurações geraram uma tabela e desta, diagramas de dispersão que, pelo método dos mínimos quadrados, forneceram as retas de regressão (interpolatrizes) apresentadas na figura 1. Com a significância estatística fixada em $95 \%(\mathrm{p}<0,05)$, as interpolatrizes foram comparadas pelo test $t$ de Student para coeficientes angulares. Tanto o GSH tecidual como o sorológico, bem como a Gli e a BT, estão apresentados em três gráficos tipo coluna vertical, com a altura refletindo a média e uma antena na respectiva coluna representando o erro padrão da média (E.P.M.), cada gráfico referente a um dos tempos T1, T2 e T3. Tais gráficos foram obtidos utilizando o soft GraphPad Prism® versão 4.0 de Fevereiro de 2005 (GraphPad Software San Diego, U.S.A.) com as comparações pelo teste comparativo de Dunnett. 


\section{RESULTADOS}

A figura 1 exibe a evolução do crescimento dos lobos residuais pós-HP e há diferença estatisticamente significante entre G1 e G2.

No que se refere à GSH, Gli e BT, somente os gráficos dos tempos que apresentaram diferença significante e não são repetitivos estão apresentados. GSH hepático e plasmático aumentaram de forma significante $(\mathrm{p}<0,01 \mathrm{em} \mathrm{T} 1 \mathrm{e}$ T2, $\mathrm{p}<0,05$ em T3) em todos os tempos: as figuras 2 e 3 exibem, respectivamente, o fato no tempo T2.

A BT não apresentou qualquer alteração significante em qualquer dos tempos. A glicemia pós-hepatectomia mostrou-se significativamente maior $(\mathrm{p}<0,01)$ em T2 e T3 (Figura 4 mostra o tempo T2).

\section{DISCUSSÃO}

O rato foi o animal de experimentação escolhido por ser o mamífero mais estudado no fenômeno da regeneração hepática $^{18}$, ter baixo custo tanto de aquisição como de manutenção e apresentar elevada resistência à infecção e ao trauma cirúrgico $^{19}$. Optou-se por animais jovens visto que a idade pode comprometer a resposta regenerativa pós-hepatectomia parcial $^{20}$ e a escolha foi por sexo masculino visando atenuar a reconhecida influência dos estrógenos na regeneração hepática $^{21}$. O período de quatorze dias entre a hepatectomia parcial e o sacrifício dos últimos animais (tempo T3) foi estabelecido com base nas evidências experimentais que a regeneração hepática pós-HP nos ratos se completa em, no máximo, duas semanas, podendo inclusive ocorrer muito antes ${ }^{2,18}$. A decisão de liberar a alimentação e a água no pré-operatório repousou nos trabalhos que sustentam que o jejum anterior aos procedimentos cirúrgicos aumentam o estresse oxidativo em

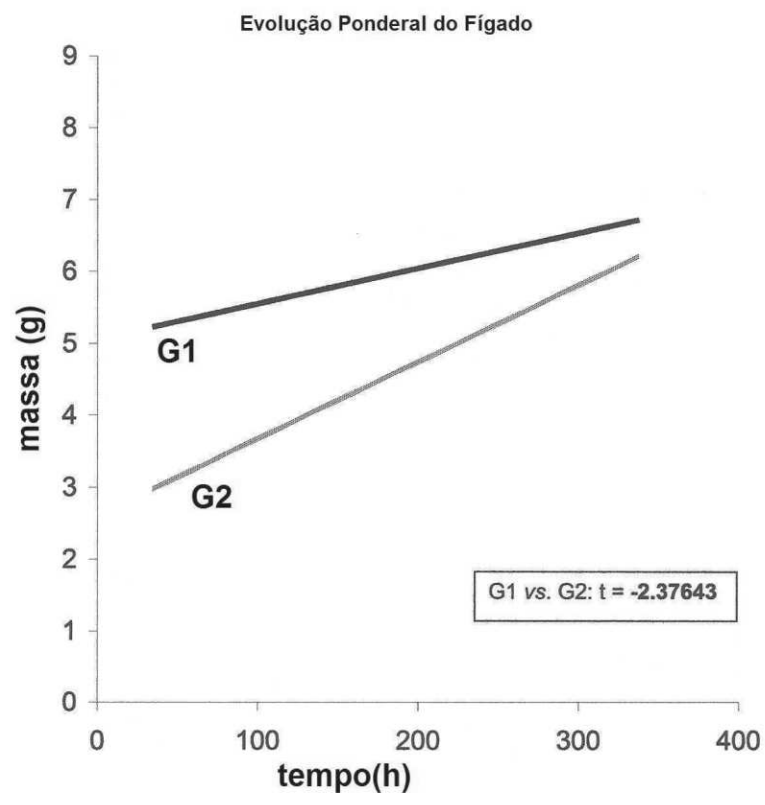

Figura 1 - Há diferença significante $(p<0,05)$ no crescimento da massa hepática entre os grupos controle G1 e experimento G2. (Teste $t$ de Student para coeficientes angulares). ratos Wistar adultos ${ }^{22}$. Apesar de que a anestesia inalatória com vapores de éter dietílico, método utilizado nas intervenções cirúrgicas realizadas, resultar em aumento da peroxidação lipídica - de forma independente e aditiva ao fator jejum ${ }^{22}-\mathrm{e}$ conseqüentemente, ser ela própria fator incentivador de

GSH no Fígado (T2)

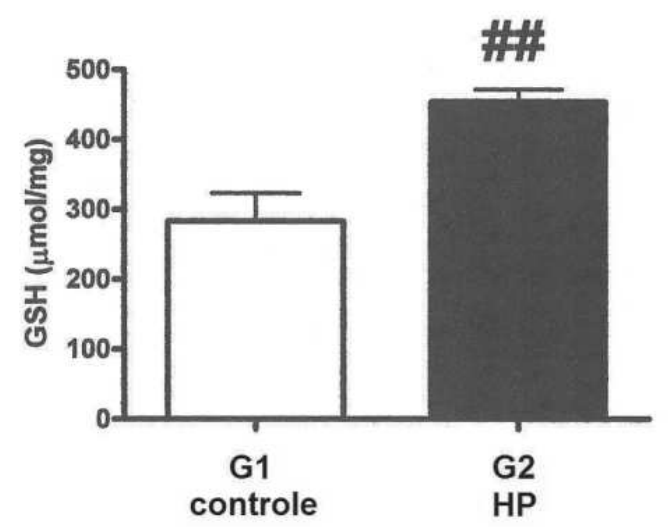

Figura 2 - Ratos parcialmente hepatectomizados exibem glutationa hepática significativamente aumentada $(p<0,01)$. Teste de Dunnett.

\section{GSH no Sangue (T2)}

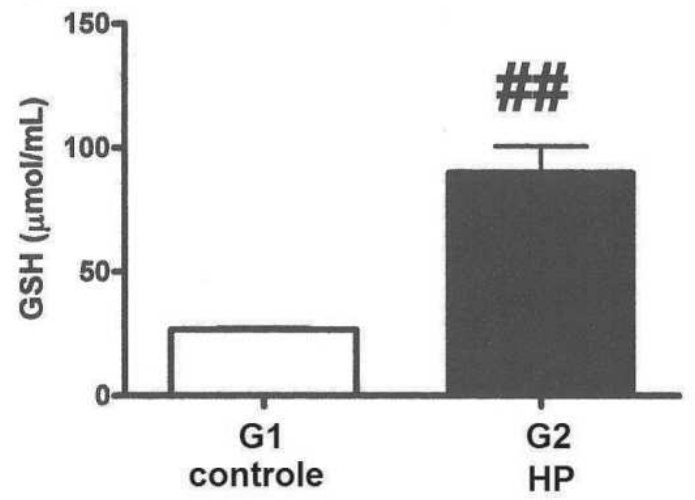

Figura 3 - Ratos parcialmente hepatectomizados mostram glutationa sangüínea significativamente aumentada $168 \mathrm{~h}$ (T2) após intervenção cirúrgica. Teste de Dunnett.

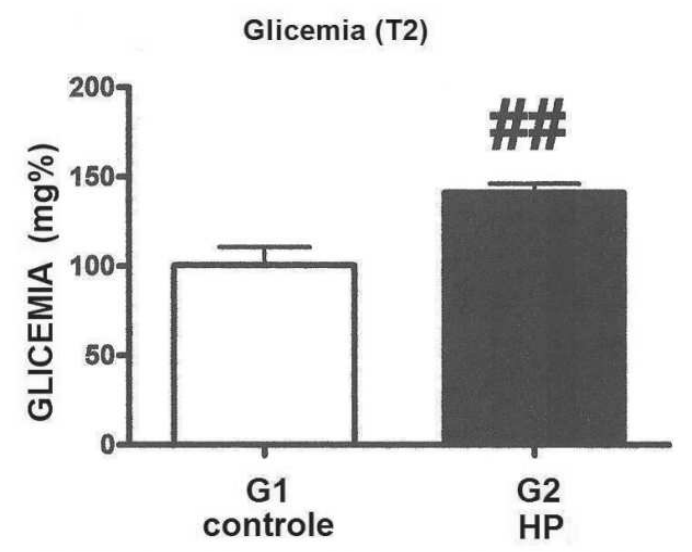

Figura 4 - Ratos parcialmente hepatectomizados exibem significante ( $p<0,01)$ hiperglicemia $168 h$ (T2) após intervenção cirúrgica. Teste de Dunnett. 
estresse oxidativo, tal variável foi minimizada pelo pequeno período do ato cirúrgico (cinco minutos, em média) e ser constituinte universal nos dois grupos, experimental e controle (G2 e G1, respectivamente).

A evolução ponderal do fígado residual pós-HP Grupo G2 - apresentou crescimento significante na comparação com G1 (Figura 1), o que é um resultado esperado visto que, em G1 não há, em princípio, qualquer fenômeno regenerativo. O coeficiente angular em G1 não é nulo porque há um crescimento hepático natural, pequeno, comum a animais jovens em desenvolvimento.

Via de regra, as células reagem ao estresse oxidativo com um aumento em seu conteúdo de glutationa como parte de sua resposta adaptativa à potencial lesão oxidativa. Aqui a GSH aumentou de modo significativo no grupo experimento, mostrando a existência de maior estresse oxidativo neste grupo. A oferta de GSH exógena, no entanto, influi negativamente sobre a evolução da regeneração hepática ${ }^{23}$. Mais ainda: os antioxidantes, de um modo geral, inibem a regeneração hepática ${ }^{6,14,24}$.

Mesmo sob regeneração, os estudos são basicamente unânimes em mostrar que a maioria, senão todas, das funções hepáticas necessárias à homeostase orgânica, incluindo sín- tese de albumina, de fatores de coagulação e de bile, manutenção da glicemia e da atividade do ciclo da uréia, permanecem preservadas $^{21,25}$. De fato, neste experimento, a bilirrubinemia total não apresentou qualquer distúrbio estatisticamente significante. No que se refere à glicemia, no entanto, significante aumento ocorreu no grupo teste. Tal hiperglicemia reflete principalmente a queda da concentração de insulina e do concomitante aumento do nível de glucagon, já experimentalmente comprovados ${ }^{26}$,após hepatectomias parciais.

A regeneração hepática é um fenômeno complexo. Muitos órgãos exibem crescimento após injúria, geralmente por mudanças hipertróficas sem aumento expressivo da população celular. O fígado é único em sua habilidade de apresentar um fenômeno essencialmente hiperplásico , com recomposição de células e tecidos. A peroxidação lipídica induzida pela HP provavelmente tem um papel neste processo de resposta hiperplásica compensatória ${ }^{27}$. Embora nosso estudo evidencie a ocorrência de um aumento do estresse oxidativo durante a regeneração hepática após hepatectomia parcial, a investigação do estresse oxidativo na regeneração hepática ainda tem resultados escassos e seu preciso papel aguarda elucidação $0^{5,24}$.

\begin{abstract}
Background: The purpose of this study was to evaluate the oxidative stress and the hepatic function on rat liver regeneration after partial hepatectomy (PH). Methods: Thirty-six young male Wistar rats were randomly assigned in two groups of 18 animals each: control group $1(G 1)$ the animals were only laparotomized, and group $2(G 2)$ rats were submitted to PH. In each group, at 36 hours (T1), 168 hours (T2) and 336 hours (T3) post-PH, a subgroup of 6 rats was chosen in a randomized way to be submitted to a total hepatectomy $(\mathrm{TH})$ in $\mathrm{Gl}$, and to complete the hepatectomy $(\mathrm{CH})$ in $\mathrm{G} 2$ rats. We measured blood samples and the residual liver lobes for reduced glutathione (GSH) (blood plasma and liver), blood concentrations of glucose (Glu), and total bilirubin (TB). All surgical procedures were performed under inhalator ether anesthesia. Results: There was no significant difference on the liver regeneration between the 2 groups. Liver and blood GSH concentrations from G2 were significantly higher than G1. Significant hyperglycemia occurred at T2 and T3 in G2. TB measurements did not show any significant difference on both groups. Conclusion: There is an increased oxidative stress in rats on liver regeneration post-PH, but the residual liver lobes did not compromise with the organic homeostasis.
\end{abstract}

Key words: Hepatectomy; Oxidative stress; Rats; Liver regeneration; Lipid peroxidation; Glutathione.

\section{REFERÊNCIAS}

1. Kountouras J, Boura P, Lygidakis NJ. Liver regeneration after hepatectomy. Hepatogastroenterology. 2001;48(38):556-62.

2. Higgins GM, Anderson RM. Experimental pathology of the liver: I. Restoration of the liver of the white rat following partial surgical removal. Arch Pathol. 1931; 12:186-212.

3. Hockings PD, Roberts T, Campbell SP, Reid DG, Greenhill RW, Polley SR, Nelson P, Bertram TA, Kramer K. Longitudinal magnetic resonance imaging quantitation of rat liver regeneration after partial hepatectomy. Toxicol Path. 2002; 30(5):606-10.

4. Minuk GY. Hepatic Regeneration: if ain't broke, don't fix it. Can J Gastroenterol. 2003;17(7):418-24.

5. Ronco MT, deAlvarez ML, Monti J, Carrillo MC, Pisani G, Lugano MC, Carnovale CE. Modulation of balance between apoptosis and proliferation by lipid peroxidation (LPO) during rat liver regeneration. Mol Med. 2002; 8(12):808-17.

6. Trejo-Solis C, Sánchez VC, Aranda-Frausro A, Sánchez-Sevilla L, Gómez-Ruíz C, Hernández-Muñoz R. Inhibitory effect of vitamin $\mathrm{E}$ administration on the progression of liver regeneration induced by partial hepatectomy in rats. Lab Invest. 2003;83(11):1669-79.

7. Ok E, Yilmaz Z, Karakücük I, Akgün H, Sahin H. Use of live oil based emulsions as an alternative to soybean oil based emulsions in total parenteral nutrition and their effects on liver regeneration following hepatic resection in rats. Ann Nutr Metab. 2003;47(5):221-7.

8. Fang YZ, Yang S, Wu G. Free radicals, antioxidants, and nutrition. Nutrition. 2002;18(10):872-9.

9. Kohen R, Nyska A. Oxidation of biological systems: oxidative stress phenomena, antioxidants, redox reactions, and methods for their quantification. Toxicol Pathol. 2002;30(6):620-50.

10. Vannucchi H, Iglesias ACRG, Morandi MV. Determinação de radicais livres em ensaios biológicos. In: Castro e Silva Jr. O, Zucoloto S, Beer Jr. A, editores. Modelos experimentais de pesquisa em cirurgia. São Paulo: Robe Editorial; 1998. p. 159-72.

11. Storey KB. Oxidative stress: animal adaptations in nature. Braz J Med Biol Res. 1996;29(12):1715-33.

12. Fridovich I. Superoxide dismutases. Adv Enzymol Relat Areas Mol Biol. 1986; 58:61-97. 
13. Meister A. Mechanism and regulation of the glutamine-dependent carbamyl phosphate synthetase of Escherichia coli. Adv Enzymol Relat Areas Mol Biol. 1989;62:315-74.

14. Melo JUS. Ácidos omega-3 e ômega-6, dimetilsulfóxido e ternatina na regeneração hepática e no estresse oxidativo em ratos [Dissertação]. Fortaleza (CE): Universidade Federal do Ceará; 2006.

15. Sedlak J, Lindsay RH. Estimation of total, protein-bound, nonprotein sulfhydril groups in tissue with Ellman's reagent. Anal Biochem. 1968;25(1):192-205.

16. Meites S. Bilirubin, direct reacting and total, modified MalloryEvelyn method. In: Faulkner WR, Meites S, editors. Selected methods of clinical chemistry. Washington: American Association for Clinical Chemistry; 1982. v. 9. p. 119-25.

17. Slein apud Vasconcelos PRL. Hepatic metabolism during sepsis [Dissertation]. Oxford: Oxford University; 1987.

18. Ramalho FS, Ramalho LNZ, Zucoloto S, Castro e Silva Jr. O. Regeneração hepática. In: Castro e Silva Jr. O, Zucoloto S, Beer Jr. A, editores. Modelos experimentais de pesquisa em cirurgia. São Paulo Robe Editorial; 1998. p. 243-58.

19. Festing MFW. Suitability of the rat for different investigations. In: Altman PI, Katz DD, editors. Inbred and genetically defined strains of laboratory animals. Part 1: mouse and rat. Bethesda: Fed Am Soc Exp Biol; 1979. p. 237-8.

20. Bucher NLR, Swaffield MN, Troia JF. The influence of age upon the incorporation of thymidine-2- $\mathrm{C}^{14}$ into the DNA of regenerating rat liver. Cancer Res. 1964;24:509-12.

21. Francavilla A, Polimeno L, DiLeo A, Barone M, Ove P, Coetzee $\mathrm{M}$, et al. The effect of estrogen and tamoxifen on hepatocyte proliferation in vivo and in vitro. Hepatology. 1989;9(4):614-20.

22. Liu PT, Kentish PA, Symons AM, Parke DV. The effects of ether anaesthesia on oxidative stress in rats - dose response. Toxicololy. 1993;80(1):37-49.
23. Holecek M, Skopec F, Sprongl L. Influence of buthionine sulfoximine, S-adenosylmethionine and glutathione on liver regeneration following partial hepatectomy. Arzneimittelforschung. 2000;50(12):1093-8.

24. Kirimlioglu V, Kirimlioglu H, Yilmaz S, Ozgor D, Coban S, Karadag N, Yologlu S. Effect of fish oil, olive oil, and vitamin E on liver pathology, cell proliferation, and antioxidant defense system in rats subject to partial hepatectomy. Transplant Proc. 2006;38(2):564-7.

25. Holecek M. Nutritional modulation of liver regeneration by carbohydrates, lipids, and amino acids: a review. Nutrition. 1999;15(10):784-8.

26. Bucher ML, Swaffield MN. Regulation of hepatic regeneration in rats by synergistic action of insulin and glucagon. Proc Natl Acad Sci U S A. 1975;72(3):1157-60.

27. Kurir TT, Markotic A, Katalinic V, Bozanic D, Cikes V, Zemunik $\mathrm{T}$, et al. Effect of hyperbaric oxygenation on the regeneration of the liver after partial hepatectomy in rats. Braz J Med Biol Res. 2004;37(8):1231-7.

Como citar este artigo:

Souza Melo JU, Campos Jr. MM, Santos JM, Barreto MV, Kimura OS. O estresse oxidativo na regeneração hepática em ratos. Rev Col Bras Cir. [periódico na Internet] 2008; 35(4). Disponível em URL: http://www.scielo.br/rcbc

Endereço para correspondência:

José Ulisses de Souza Melo

R.Afonso Celso,897 - Aldeota

60140-190 - Fortaleza - CE

E-mail:dr.ulisses@ufc.br 